\title{
Thoracoscopic Surgical Navigation System for Cancer Localization in Collapsed Lung Based on Estimation of Lung Deformation
}

\author{
Masahiko Nakamoto $^{1}$, Naoki Aburaya ${ }^{1}$, Yoshinobu Sato ${ }^{1}$, Kozo Konishi ${ }^{2}$, \\ Ichiro Yoshino $^{2}$, Makoto Hashizume ${ }^{2}$, and Shinichi Tamura ${ }^{1}$ \\ ${ }^{1}$ Division of Image Analysis, Graduate School of Medicine, Osaka University, Japan \\ ${ }^{2}$ Graduate School of Medical Sciences, Kyushu University, Japan
}

\begin{abstract}
We have developed a thoracoscopic surgical navigation system for lung cancer localization. In our system, the thoracic cage and mediastinum are localized using rigid registration between the intraoperatively digitized surface points and the preoperative CT surface model, and then the lung deformation field is estimated using nonrigid registration between the registered and digitized point datasets on the collapsed lung surface and the preoperative CT lung surface model to predict cancer locations. In this paper, improved methods on key components of the system are investigated to realize clinically acceptable usability and accuracy. Firstly, we implement a non-contact surface digitizer under thoracoscopic control using an optically tracked laser pointer. Secondly, we establish a rigid registration protocol which minimizes the influence of the deformation in different patient's positions by analyzing MR images of volunteers. These techniques were evaluated by in vitro and clinical experiments.
\end{abstract}

\section{Introduction}

The detection ratio of early small lung cancers has been improved by CT screening, and then resection of the small cancers by thoracoscopic surgery has recently become common as a minimally invasive technique. However, one problem is that localization of a small cancer often takes long time and sometimes even results in failure under thoracoscopic view. The lung is greatly deformed due to lung collapse by air suction to create a sufficient amount of workspace for surgical operation (Fig. 11). Thus the cancer position may change largely from its original position in a preoperative CT image. Furthermore, weak tactile feedback of surgical instruments makes the cancer localization difficult. Therefore, in order to narrow the extent of the existence possibility of a cancer in the collapsed lung, a system which predicts and indicates the cancer position during the surgery is highly desirable.

To assist cancer localization, Shimada et al. developed a magnetic cancer tracking system [1. In this system, a small magnetic marker is embedded near the tumor by CT-guided bronchoscopy just before the surgery, and then the

N. Ayache, S. Ourselin, A. Maeder (Eds.): MICCAI 2007, Part II, LNCS 4792, pp. 6876, 2007.

(C) Springer-Verlag Berlin Heidelberg 2007 


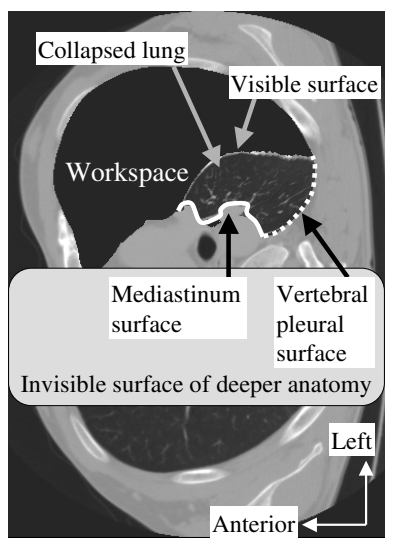

Fig. 1. Axial section of lung after collapse in the lateral position

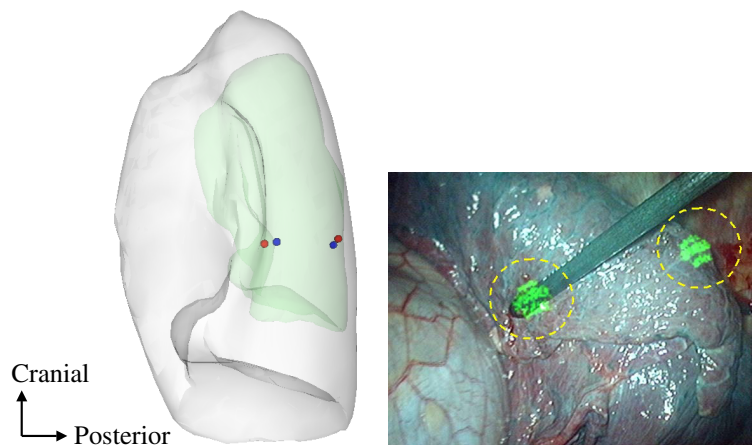

(a) Estimated lung shape (b) AR thoracoscopic imand cancer positions. age. Yellow circles: superimBlue points: actual po- posed cancers.

sitions (gold standard).

Red points: estimated

positions.

Fig. 2. Results of preliminary clinical experiment

tumor can be localized by tracking the embedded marker during the surgery. However, this approach requires an additional intervention procedure before the surgery.

As an alternative approach that does not involve additional intervention, we have developed a surgical navigation system for cancer localization which estimates the collapsed lung deformation during the surgery 2]. In this system, the thoracic cage and mediastinum are localized using rigid registration between the digitized chest skin surface points and preoperative CT skin surface model, and then the lung deformation is estimated using nonrigid registration between the digitized and registered collapsed lung surface points and preoperative CT lung surface model.

In our previous work [2, however, there are the following problems. (1) Physical contact of a long digitizing probe with the collapsed lung surface was necessary to acquire the 3D surface position data of the lung surface. (2) Difference in the patient's position (supine during CT scanning and lateral during the surgery) was not considered in the rigid registration to localize the thoracic cage and mediastinum during the surgery. The former problem may cause risk of damaging the lung as well as degradation of positional accuracy due to surface deformation at a contact point or slight bend of the long probe. The latter may cause significant localization errors for the thoracic cage and mediastinum during the surgery due to skin deformation in different patient's positions, which will finally affect cancer localization accuracy.

In this paper, we describe improved methods which address the above mentioned problems. In order to solve the problems, we aim at the followings. 
(1) Implementation and test of a non-contact surface digitizer that is compatible with thoracoscopy. (2) Establishment of the rigid registration protocol which minimizes the influence of the different patient's positions. Previous studies investigated the use of laser systems for non-contact organ surface digitizing 3] 4] 5. However, a dedicated endoscope in which a laser pointer is embedded, as described in [3], is not widely used, and a laser-scanner used in [5] is incompatible with thoracoscopy. In contrast, we combine a conventional laser pointer and thoracoscope both of which are tracked by an optical tracker so as to be widely available and compatible with thoracoscopy. To develop the rigid registration protocol, MR images of several volunteers in the supine and lateral positions are analyzed. The protocol is derived from the analysis results.

\section{Methods}

\subsection{System Overview}

The thoracoscopic surgical navigation system consists of an optical 3D tracker (Polaris, Northern Digital Inc., Canada), an oblique-viewing thoracoscope, a 3D position digitizer (i.e. stylus probe tracked by the optical 3D tracker), a laser pointer (KOKUYO, IC-GREEN, Japan), and a PC (Xeon $3.0 \mathrm{GHz} \times 2,2 \mathrm{~GB}$ memory). Optical markers are attached to the thoracoscope, 3D position digitizer, and laser pointer so that their position and orientation are measured by the

optical tracker. We denote their position and orientation as $T_{\text {scope }}, T_{\text {digitizer }}$ and $T_{\text {laser }}$, respectively. $T$ is a $4 \times 4$ matrix representing rigid transformation defined by $3 \times 3$ rotation matrix $R$ and translational $3 \mathrm{D}$ vector $\mathbf{t}$. The oblique-viewing thoracoscope camera is calibrated by the method described in [6] beforehand.

In this system [2], the thoracic cage and mediastinum are localized using ICP rigid registration between the digitized chest skin surface points and preoperative CT skin surface model, and then deformation field due to lung collapse is estimated by point-based nonrigid registration [7]. The preoperative CT image is deformed by the obtained deformation field, which is the continuous and smooth in the whole CT space, and then the collapsed lung shape and cancer position are estimated (Fig. 2(a)). The estimated cancer position is superimposed onto live thoracoscopic images, so that a surgeon can find a cancer from the neighborhood of the indicated position (Fig. 2(b) . In the preliminary clinical experiments, the estimation accuracy of the cancer positions were around $10 \mathrm{~mm}$. The acquisition time of the digitized points was 5 minutes. Computation time for estimation of lung deformation was also 5 minutes. These results showed that potential usefulness of the system for narrowing the extent of the existence possibility of the cancer. Although the feasibility of our approach was confirmed, we also have found that accuracy was sometimes unstable (for examples, $20 \mathrm{~mm}$ or more), and thus the problems described in the previous section were clarified.

\subsection{Digitizing Surface Points by Laser Pointer}

To realize non-contact surface digitizing, we employed a commercially available laser pointer. While scanning the target surface using the laser pointer by freehand, 


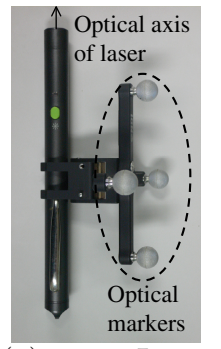

(a) pointer.

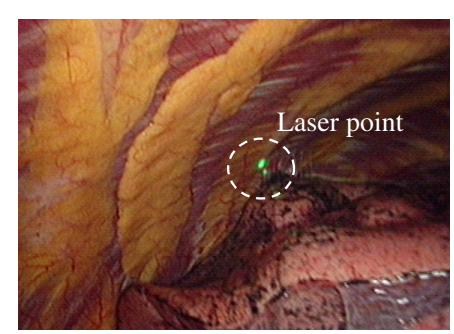

(b) Original image.

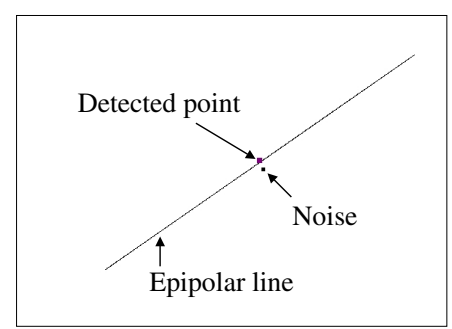

(c) Detected point.

Fig. 3. Detection of laser point from thoracoscopic image

the laser points on the surface are imaged by thoracoscope. Thoracoscopic images, $T_{\text {laser }}$, and $T_{\text {scope }}$ are recorded simultaneously during scanning, and then image processing for $3 \mathrm{D}$ coordinates measurement described below is performed in real-time.

Figure 3 shows the appearance of the laser pointer and an example of laser point detection. We employ a green laser to obtain high contrast between the laser point and the organ surface. To detect the laser point region in the thoracoscopic image, color space conversion is performed and then the candidate regions are detected by p-tile thresholding. The center of gravity of each region is calculated, and then the region that is closest to the epipolar line is selected as the laser point.

The $3 \mathrm{D}$ line passing through the focal point and the detected point, $\mathbf{l}_{\text {view }}$, is written as $\mathbf{l}_{\text {view }}=s T_{\text {scope }}\left(\left(p_{x}-c_{x}\right) / f_{x},\left(p_{y}-c_{y}\right) / f_{y}, 1.0\right)^{T}+\mathbf{t}_{\text {scope }}$, where $s$ is an arbitrary scalar, and $\left(p_{x}, p_{y}\right)$ is position of the detected point. $\left(c_{x}, c_{y}\right)$ and $\left(f_{x}, f_{y}\right)$ are image center and focal length, respectively. The 3D line of the laser, $\mathbf{l}_{\text {laser }}$, is written as $\mathbf{l}_{\text {laser }}=s R_{\text {laser }} \mathbf{v}_{\text {laser }}+T_{\text {laser }} \mathbf{q}_{\text {laser }}$, where $\mathbf{v}_{\text {laser }}$ and $\mathbf{q}_{\text {laser }}$ are respectively the direction and position defining the laser line relative to the attached optical markers, which are obtained during calibration stage. The 3D position of the laser point is defined as the intersection point of $\mathbf{l}_{\text {view }}$ and $\mathbf{l}_{\text {laser }}$.

\subsection{Rigid Registration of Preoperative CT Model}

Rigidity evaluation due to patient's position change using MR images of volunteers. While the visible surface of the collapsed lung is digitized during surgery using the laser pointing system, subsequent nonrigid registration will be unstable without positional information on the invisible surface of deeper anatomy (invisible surface) attached to the mediastinum and the thoracic cage around the vertebra (see Fig. 11). In our previous system [2, the invisible surface is localized using rigid registration between actual patient and preoperative skin surface model assuming rigid relation between the invisible and skin surfaces. However, patient's position changes from supine position during preoperative CT scanning to lateral position during surgery, and then the rigidity between the invisible and skin surfaces may not be kept after the position change. 
We analyzed MR images of 6 volunteers to evaluate the rigidity. MR images of the same subject taken in lateral and supine positions were registered using regions around vertebral column by supposing that the thoracic cage is rigid and stable around the vertebral column in spite of the position change. Figure 4 shows axial and coronal sections of the registered images. Although misalignment over the skin, diaphragm, and mediastinal surfaces were around $10 \mathrm{~mm}$ or more due to deformation, misalignment over a wide range of backside of the thoracic cage as well as around the vertebral column (i.e. region enclosed with rectangle in Fig. 4(a) was around $2 \mathrm{~mm}$. As a result of observation of 6 subjects, the following findings were obtained: (1) A wide range of backside of the thoracic cage kept the rigidity with the invisible surface around the vertebral column (Hereafter, we call it the vertebral pleural surface). (2) The chest skin largely deformed due to the position change and did not keep rigidity with the invisible surface. (3) The median line on the sternum largely moved due to the position change but it was only along posterior-anterior direction in the median plane.

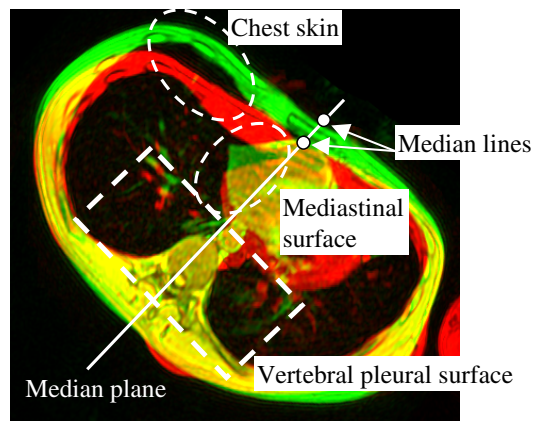

(a) Axial section.

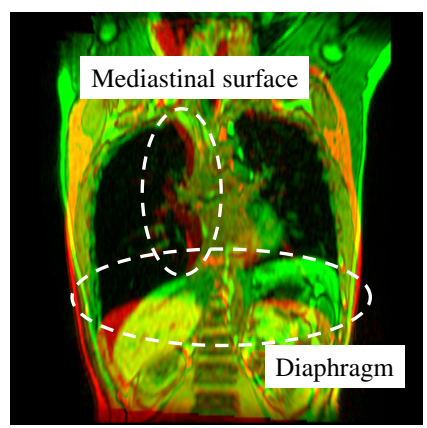

(b) Coronal section.

Fig. 4. Deformation caused by position change. Green: lateral position. Red: supine position.

Proposed registration protocol. Based on the findings described above, we derive the following rigid registration protocol. (1) Points on backside of the thoracic cage, which are intraoperatively acquired using the laser pointing system under thoracoscope, are registered to the preoperative CT lung surface. (2) Points on the skin along the median line, which are acquired using a 3D position digitizer, are registered to the preoperative CT median plane. Surface model of the thoracic cage $S_{t c}$ and the median plane $S_{m p}$ are reconstructed from patient's CT image. The points on the skin along the median line $\left\{\mathbf{q}_{i}\right\}$ were acquired and then digitized surface points on the thoracic cage $\left\{\mathbf{p}_{i}\right\}$ were acquired after collapse under thoracoscope. ICP algorithm is performed by minimizing the following cost function: 


$$
E\left(T_{\text {rigid }}\right)=\sum_{i=1}^{M}\left|d\left(S_{t c}, T_{\text {rigid }} \mathbf{p}_{i}\right)\right|^{2}+\sum_{j=1}^{N}\left|d\left(S_{m p}, T_{\text {rigid }} \mathbf{q}_{j}\right)\right|^{2},
$$

where $T_{\text {rigid }}$ is a rigid transformation from the patient space to the CT space. $d(S, \mathbf{p})$ is a function representing the closest distance between a surface $S$ and a $3 \mathrm{D}$ position $\mathbf{p}$. Using estimated $T_{\text {rigid }}$, the vertebral pleural surface is located.

\section{$3 \quad$ Experimental Results}

\subsection{Evaluation of Laser Surface Digitizing}

Laboratory experiments. We compared accuracy of digitized surface points by the laser pointer with that by the conventional digitizing probe. The phantom was fixed in the training box, and then its surface points were acquired by the laser pointer and digitizing probe through a trocar under thoracoscopic control. The error was defined as an average distance between the digitized surface points and the gold standard surface. The gold standard surface was determined by ICP registration between densely acquired surface points and the surface model reconstructed from $\mathrm{CT}$ images of the phantom. The errors of the laser and probe digitizing were $3.6 \pm 1.4 \mathrm{~mm}$ and $4.6 \pm 2.0 \mathrm{~mm}$, respectively, and thus the laser pointer was more accurate than the probe.

Clinical experiments. We tested the laser pointing surface digitizer under thoracoscopic control for a real patient according to the IRB approved protocol. The conventional long probe were also employed to compare with the laser pointing digitizer. Using the non-contact digitizer, 90 points were acquired from 136 measurements. 21 measurements were failed to detect the laser points, and 15 measurements were rejected due to small parallax (less than 20 degrees) between the optical axis of the laser and the line of sight to the laser point. Average parallax was 34 degrees and acquisition time was around 4 minutes. Using the long probe, 53 points were acquired and acquisition time was around 3 minutes. Figure 5 shows distribution of acquired points. The extents of point datasets acquired by the two digitizers were comparable.

\subsection{Simulation Experiments Using Volunteer MR Images to Validate Rigid Registration}

The proposed rigid registration protocol was compared with intensity-based rigid registration and conventional ICP algorithm using the skin surface. The results of intensity-based method is regarded as ideal since rich information inside the body is available, which is unable to acquire without intraoperative MR or CT.

Rigid registration of MR images between in the supine position at inspiration and in the lateral position at expiration was performed for six pairs of MR images by using the three protocols. Around 10 and 40 points were acquired from the median line and the backside of the thoracic cage, respectively. Registration error $E_{r e g}$ of estimated rigid transform $T_{\text {rigid }}$ was defined 


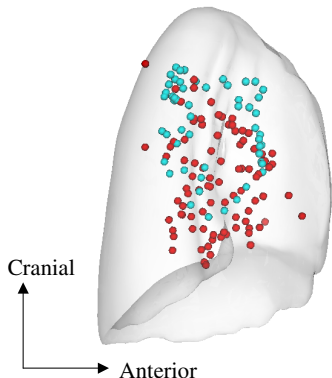

(a) Lateral view.

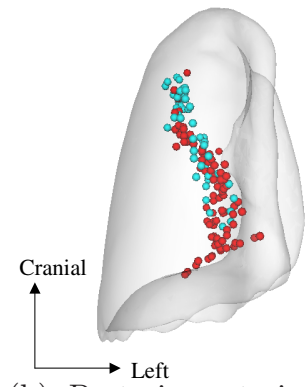

(b) Posterior-anterior view.

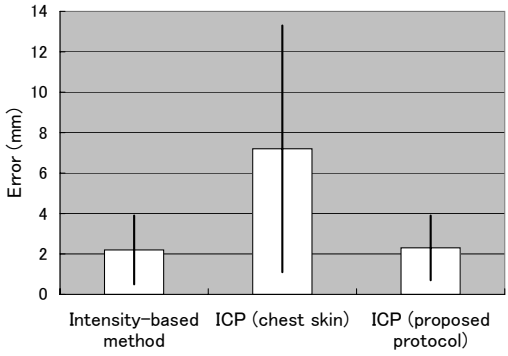

Fig. 5. Results of clinical experiment for lung Fig. 6. Registration error of vertesurface digitizing. Red points: acquired by laser bral pleural surface pointer. Blue points: acquired by digitizing probe.

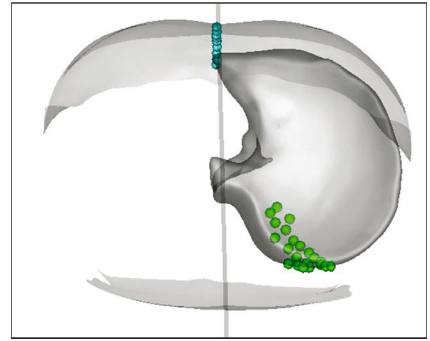

(a) Cranial-caudal view.

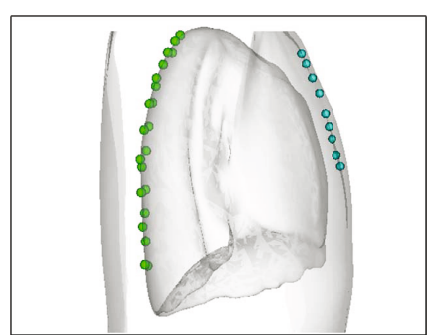

(b) Lateral view.

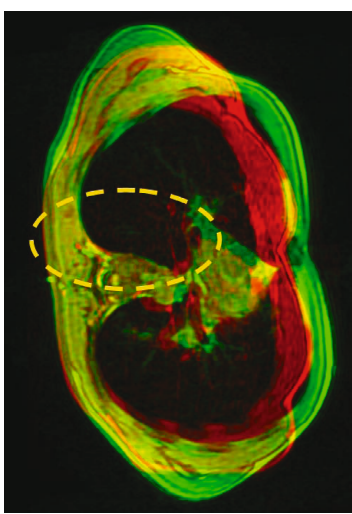

(c) Intensity-based.

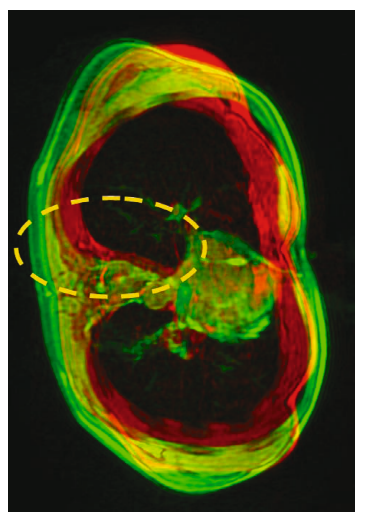

(d) ICP (chest skin).

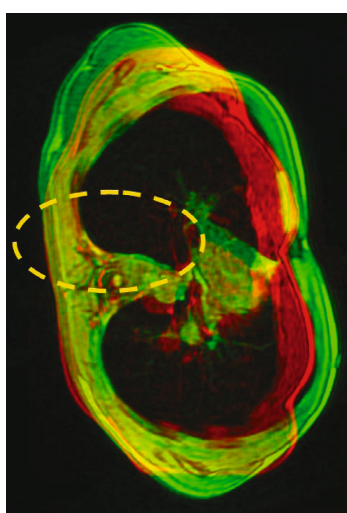

(e) ICP (proposed protocol).

Fig. 7. Results on accuracy evaluation for rigid registration. Blue and green points for rigid registration in the upper images are acquired from the median line and the backside of the thoracic cage, respectively. Lower images are axial sections of registered images in lateral position. Green: lateral position. Red: supine position. 
as $E_{\text {reg }}=1 / N \sum_{i=1}^{N}\left|d\left(S, T_{\text {rigid }} \mathbf{q}_{i}\right)\right|$, where $\left\{\mathbf{q}_{i}, i=1, \ldots, N\right\}$ is a point set acquired from the vertebral pleural surface in the lateral position, and $S$ is the vertebral pleural surface in the supine position. As a result, the error of the proposed method was $2.3 \pm 1.6 \mathrm{~mm}$ and it was comparable with the error of the intensity-based method (Fig. 6). The error of ICP algorithm using the skin was around $7 \mathrm{~mm}$ due to the large deformation caused by the position change. Figure 7 shows axial sections of the registered MR images. In the cases of the intensity-based and the proposed method, it was confirmed that misalignment on the vertebral pleural surface was sufficiently small.

\section{Discussion and Conclusions}

We have described improved methods for key components of our thoracoscopic surgical navigation system for cancer localization. We implemented a non-contact surface digitizer using a conventional laser pointer and thoracoscope. In the experiments, accuracy of the non-contact digitizer was better than that of the conventional long digitizing probe, and clinical feasibility was confirmed. As a result, the extent of measured points acquired by the non-contact digitizer was comparable to that of the conventional probe. According to the surgeon's opinion, the non-contact digitizer was preferred since it allowed him to digitize lung surface without a special care for not damaging the lung by the conventional probe. We also established rigid registration protocol based on the evaluation using MR images of volunteers. In the simulation experiment, registration error of the proposed method was around $2 \mathrm{~mm}$ and it was comparable with that of the intensity-based method. Since the error of rigid registration affects accuracy of lung deformation estimation, the proposed method will improve overall accuracy of the system. Although evaluation of the system incorporating the non-contact digitizer and the proposed registration protocol currently have not been performed yet, improvements of the accuracy and usability of the system can be expected if they were employed.

Future work includes validation study of the whole system incorporating the proposed methods and integration with biomechanical deformation model of the collapsed lung. Point datasets on the collapsed lung surface acquired by our techniques will utilized as constraints to estimate the biomechanical lung deformation. Our system can be used as a platform of the development and application of such biomechanical techniques.

\section{References}

1. Shimada, J., et al.: Intraoperative magnetic navigation system for thoracoscopic surgery and its application to partial resection of the pig lang. In: CARS 2004. Computer Assisted Radiology and Surgery: 18th International Congress and Exhibition, pp. 437-442 (2004)

2. Nakamoto, M., et al.: Estimation of intraoperative lung deformation for computer assisted thoracoscopic surgery. Int. J. Computer Assisted Radiology and Surgery 1(suppl. 1), 273-275 (2006) 
3. Nakamura, Y., et al.: Laser-pointing endoscope system for natural 3D interface between robotic equipments and surgeons. Studies in health technology and informatics 81, 348-354 (2001)

4. Krupa, A., et al.: Autonomous 3-D positioning of surgical instruments in robotized laparoscopic surgery using visual servoing. IEEE Transactions on Robotics and Automation 19, 842-853 (2003)

5. Sinha, T.K., et al.: A method to track cortical surface deformations using a laser range scanner. IEEE Transactions on Medical Imaging 24, 767-781 (2005)

6. Yamaguchi, T., et al.: Development of camera model and calibration procedure for oblique-viewing endoscopes. Computer Aided Surgery 9, 203-214 (2004)

7. Chui, H., et al.: A unified non-rigid feature registration method for brain mapping. Medical Image Analysis 7, 113-130 (2003) 